\title{
BMJ Open Protocol for the Lactoferrin Infant Feeding Trial (LIFT): a randomised trial of adding lactoferrin to the feeds of very-low birthweight babies prior to hospital discharge
}

Andrew Martin, ${ }^{1}$ Alpana Ghadge, ${ }^{1}$ Paolo Manzoni, ${ }^{2}$ Kei Lui, ${ }^{3,4}$ Rebecca Brown, ${ }^{1}$ William Tarnow-Mordi, ${ }^{1}$ for the LIFT Collaborative Study Group

To cite: Martin A, Ghadge A, Manzoni P, et al. Protocol for the Lactoferrin Infant Feeding Trial (LIFT): a randomised trial of adding lactoferrin to the feeds of very-low birthweight babies prior to hospital discharge. BMJ Open 2018;8:e023044. doi:10.1136/ bmjopen-2018-023044

- Prepublication history and additional material for this paper are available online. To view these files, please visit the journal online (http://dx.doi org/10.1136/bmjopen-2018023044).

Received 19 March 2018 Revised 17 May 2018 Accepted 19 June 2018

\section{Check for updates}

(C) Author(s) (or their employer(s)) 2018. Re-use permitted under CC BY-NC. No commercial re-use. See rights and permissions. Published by BMJ.

${ }^{1}$ NHMRC Clinical Trials Centre, University of Sydney, Sydney, New South Wales, Australia ${ }^{2} \mathrm{~S}$. Anna Hospital Torino, Torino, Italy

${ }^{3}$ Department of Newborn Care Royal Hospital for Women, Randwick, New South Wales, Australia

${ }^{4}$ School of Women's and Children's Health, University of New South Wales, Kensington, New South Wales, Australia

Correspondence to Dr William Tarnow-Mordi; williamtm@med.usyd.edu.au

\section{ABSTRACT}

Introduction Very-low birthweight (VLBW, $<1500 \mathrm{~g}$ )

infants comprise about $1 \%-1.4 \%$ of all births in high-income countries. Every year, about 3000 VLBW babies in Australia and New Zealand receive intensive care. Many die or else survive with severe brain injury, retinopathy, late-onset sepsis or necrotising enterocolitis (NEC), each of which carries substantial risk of disability.

Methods and analysis This trial tests whether adding bovine lactoferrin (bLF) to feeds in VLBW infants improves (1) survival to hospital discharge free from brain injury, late-onset sepsis, NEC and treated retinopathy of prematurity (primary composite end point); (2) each component of the primary composite end point and (3) time to reach full enteral feeds, number of blood transfusions, chronic lung disease and length of hospital stay. It includes a cost-effectiveness analysis of bLF in improving survival free from major morbidity, and evaluates the effect of bLF on survival and developmental outcomes at 24 to 36 months corrected gestational age. This is a multicentre, twoarm, randomised trial comparing the treatment group receiving bLF added to breast milk or formula milk daily (up to $250 \mathrm{mg} / \mathrm{kg} /$ day bLF) versus the control group receiving no bLF supplementation. The intervention is administered until 34 completed weeks corrected gestation or for 2 weeks, whichever is longer, or until discharge home, if earlier. The target sample size of 1500 participants yields $85 \%$ power, at the twosided $5 \%$ level significance, to detect a difference in proportions meeting the primary outcome assuming the true probability is $74 \%$ in controls and $80.5 \%$ in the bLF group.

Ethics and dissemination This protocol was approved by Northern Sydney Local Human Research Ethics Committee in January 2017 (Version 2.0, Reference 1003-118M) and other relevant ethics committees. The findings of the trial will be disseminated through peer-reviewed journals and conference presentations.

Trial registration number ACTRN12611000247976; Preresults.

\section{Strengths and limitations of this study}

- This protocol is for a well-powered, blinded, randomised controlled trial (RCT) to determine if supplementing oral feeds in very-low birthweight infants with bovine lactoferrin (bLF) up to $250 \mathrm{mg} /$ $\mathrm{kg} /$ day improves the primary composite outcome of survival without major morbidity (defined as brain injury, necrotising enterocolitis, late-onset sepsis or severe retinopathy of prematurity).

- Recommended approaches for constructing the primary composite outcome were followed. Each component is clinically important (eg, the hospital morbidity end points are independently associated with later disability), amenable to unbiased assessment and corresponds to biological pathways that bLF may favourably influence.

- While the Lactoferrin Infant Feeding Trial (LIFT) is not powered to detect an improvement on survival alone or on other individual components of the primary end point alone, the Statistical Analysis Plan for includes a proposal to combine LIFT data with similar RCTs to more precisely estimate the effect of lactoferrin on survival, late-onset sepsis and necrotising enterocolitis.

\section{INTRODUCTION}

One in 10 babies, or 15 million per year, is born preterm, that is, before 37 weeks' gestation, and the number is rising. ${ }^{1}$ Very-low birthweight (VLBW $<1500 \mathrm{~g}$ ) babies account for about $1 \%$ to $1.4 \%$ of all births in high-income countries such as Australia or the USA. $^{2-4}$ Every year, about 3000 VLBW babies in Australia and New Zealand are admitted for intensive care. ${ }^{3}$ Of these, about 1200 die or survive with severe brain injury, retinopathy (ROP), late-onset sepsis or necrotising enterocolitis (NEC), each of which is associated with substantial risk of childhood disability. Survivors are at increased risk of 
poor growth; hospital admission; visual deficit; cerebral palsy and subsequent neurosensory, cognitive and behavioural impairment; attention deficit; school failure; asthma; hypertension; diabetes; obesity; autism and other psychiatric disorders. ${ }^{15-8}$ Affordable interventions that have the potential to reduce mortality and morbidity in these infants merit careful evaluation.

Lactoferrin is a cationic glycoprotein found in mammalian milk, tears, saliva, sweat, cerebrospinal fluid and neutrophils. It is present on mucosal surfaces and is part of the innate immune response. ${ }^{9}$ Plasma levels of lactoferrin are low in preterm infants, but rise in response to infection. ${ }^{1011}$

Lactoferrin is a potent inhibitor and microbicide for bacteria, fungi, viruses and protozoa. Several modes of action are described, for example, sequestering iron (an essential substrate for pathogens), disrupting bacterial and fungal cell membranes, inhibiting microbial adhesion to host cells, preventing biofilms, blocking viral entry and transmission between host cells, inhibiting protozoal growth $^{12-16}$ and promoting the growth of bifidobacteria in the gut. ${ }^{17}$

Human lactoferrin (hLF) has a molecular weight of 80 KDa and shares $77 \%$ amino acid homology with its bovine isoform, bLF. ${ }^{18}$ The activity of bLF against Gram-negative bacteria is partly mediated by its positively charged $\mathrm{N}$-terminal peptide which binds to negatively charged lipopolysaccharide in the bacterial membrane, inactivating surface anchored type III secretory system virulence proteins. ${ }^{12}$ Lactoferrin also binds to lipoteichoic acid in Gram-positive bacterial membranes. ${ }^{19}$ Lactoferrin undergoes partial acid proteolysis in the stomach to yield lactoferricins, which are peptides with enhanced antimicrobial activity. ${ }^{20}$ This may partly explain why H2 antagonists, which suppress gastric acid and proteolysis in the stomach, are associated with increased risk of sepsis and why bLF has a protective effect. ${ }^{21}$

VLBW infants are at risk of suboptimal nutrition ${ }^{22}$ owing to feed intolerance or illness, and an insufficient intake of lactoferrin may contribute to their vulnerability. Lactoferrin has anti-inflammatory activity, demonstrated by its ability to prevent gut injury in experimental colitis. ${ }^{23}$ Its bifidogenic activity in the gut ${ }^{17}$ may improve tolerance to feeds and reduce the risk of NEC. ${ }^{24}$ It is also an antioxidant that reduces free radical formation and lipid peroxidation caused by adding iron to breast milk or formula. ${ }^{25}$ bLF has no known toxicity and is registered as GRAS (Generally Recognised As Safe) by the US Food and Drug Administration ${ }^{26}$ and is a staple component of the human diet because it is present in cow's milk at a concentration of $\sim 0.4 \mathrm{mg} / \mathrm{mL} .{ }^{27}$ In rodents, the no-observed-adverse-effect level of bLF was at least $2000 \mathrm{mg} / \mathrm{kg} /$ day ${ }^{28}$ Although bLF has an excellent safety record and no allergic or adverse reactions occurred in our previous trial, ${ }^{29}$ there is a theoretical risk of cow's milk allergy in later childhood.

Supplementing standard feeds with lactoferrin may be an effective strategy to safely improve outcomes for VLBW infants. A Cochrane Review published in 2011 identified one randomised controlled trial (RCT), led by a member of our group, ${ }^{29}$ of adding a fixed daily dose of $100 \mathrm{mg}$ bovine lactoferrin (bLF) to feeds for 4 to 6 weeks in $\mathrm{n}=472$ VLBW infants. ${ }^{30}$ No trials of adding hLF to feeds in VLBW infants were published at that time. This trial estimated a statistically significant $\sim 65 \%$ relative risk reduction of late-onset sepsis, sepsis-related mortality, NEC and severe ROP, and estimated a non-significant $67 \%$ relative risk reduction of hospital mortality, and identified no safety issues. ${ }^{29}$ Despite these promising results, the earlier and current Cochrane reviews concluded that evidence of low quality suggests that lactoferrin supplementation to enteral feeds with or without probiotics decreases late-onset sepsis and NEC in preterm infants without adverse effects but further data using different doses and evaluating long-term follow-up from ongoing trials were needed. ${ }^{3031}$ In an unpublished survey in March 2012, 96\% $(22 / 23)$ of Australian and New Zealand neonatal intensive care units (NICUs) did not use bLF. The Lactoferrin Infant Feeding Trial (LIFT) was consequently designed in 2013 and initiated in June 2014 to address the uncertainty over the effectiveness and safety of bLF as a feed supplement for VLBW infants.

The aim of LIFT is to determine the effectiveness of adding bLF to the feeds of preterm babies of $<1500 \mathrm{~g}$ birth weight. The specific objectives are to determine the effect on the primary composite outcome of survival without major morbidity (defined as brain injury, NEC, late-onset sepsis or severe ROP of prematurity), as well as a series of secondary end points detailed below in Methods and analysis section.

Version 1 of the LIFT protocol was finalised in September 2013 and prospectively registered on the Australian and New Zealand Clinical Trials Registry. Version 2 of the protocol was finalised in January 2017 and updates were made to the Australian and New Zealand Clinical Trials Registry entry. A summary of the changes made for version 2 of the protocol is included as an e-supplemental 1.

\section{METHODS AND ANALYSIS}

\section{Study design and eligibility}

LIFT is a multicentre phase III trial that randomises VLBW infants from 16 NICUs from Australia $(n=14)$ and New Zealand $(n=2)$ to an experimental group receiving bLF added to breast milk or formula milk once daily, or to a control group receiving no bLF added to breast milk or formula milk.

Infants born with a birth weight of $<1500 \mathrm{~g}$ and $\leq 7$ days of age are eligible for LIFT provided they have no severe congenital anomalies likely to cause death and that a parent provides written informed consent.

The randomisation is performed, via a centralised web-based system, in a 1:1 ratio using a minimisation approach that stratifies for NICU, gender, birth weight $(<1000 \mathrm{vs} \geq 1000-1499 \mathrm{~g})$ and according to whether the infant is from a single or multiple birth. 
Any family that wishes to withdraw their baby from the trial may do so, without giving a reason and without any change in any other aspect of treatment. Parents of any baby who is withdrawn from the study after randomisation and before or after the intervention is administered, permission will be sought to follow baby's progress and allow collection of outcome data. Parents may choose to withdraw this permission as well. Parents may withdraw their consent for provision of specific information, such as economic data, while continuing to participate in the clinical study.

\section{Intervention and blinding}

Infants allocated to the treatment group receive a once daily dose of lactoferrin in breast milk or formula milk to a daily dose of up to $250 \mathrm{mg} / \mathrm{kg}$ bLF. Lactoferrin is supplied by Australia's Own Pty Ltd. This product is listed on the Australian Therapeutics Goods Register.

Infants allocated to the control group receive no bLF added to breast milk or formula milk. The allocated feeding strategy (hereafter referred to as the treatment) is intended to commence as soon as possible after randomisation and is to continue until 34 completed weeks corrected gestation or for 2 weeks, whichever is longer, or until discharge home, if earlier.

Treatment allocation is blinded. A designated person (eg, bedside nurse, pharmacist, member of milk kitchen staff or any appropriate staff member) prepares the milk feed containing either bLF (treatment group) or nothing (control group). This blinding procedure avoids use of a placebo, such as sucrose or maltose, which cannot be guaranteed to be biologically and physiologically inert in preterm or VLBW infants.

Unblinding is allowed if knowledge of investigational product is needed for treatment of a serious adverse event. In an emergency, sites shall contact the coordinating centre to obtain treatment identity from the randomisation personnel. Discussion with the chief investigator or delegate is required prior to unblinding and any instance of unblinding is fully documented.

Blinded treatment information is collected to allow adherence to be assessed. No concomitant medications or treatments are contraindicated. Concomitant medications will not be recorded during the study, except for medications being taken when a suspected unexpected serious adverse reaction (SUSAR) is encountered.

\section{End points}

The primary end point is a composite of survival to hospital discharge free from:

1. Major morbidity at 36 completed weeks corrected gestational age defined as:

- Brain injury on ultrasound or

- NEC of grade II or higher or

- Late-onset sepsis

2. ROP treated according to local guidelines (laser surgery, cryotherapy or monoclonal antibody therapy) by discharge from hospital.
Brain injury on ultrasound is defined as: grade 3 intraventricular haemorrhage (IVH) (ventricular distension with blood), grade $4 \mathrm{IVH}$ (intraparenchymal haemorrhage), echodense intraparenchymal lesions, periventricular leukomalacia, porencephalic cysts or ventriculomegaly (97 percentile plus $4 \mathrm{~mm}) .{ }^{32} 33$

NEC is defined as a proven diagnosis with all of the following three criteria:

1. At least one systemic sign (temperature instability, apnoea, bradycardia or lethargy) and at least one intestinal sign (residual of $25 \%$ of the previous feed on two consecutive occasions or abdominal distension, or vomiting or faecal blood).

2. A profile consistent with definite NEC including abdominal wall cellulitis and palpable abdominal mass or pneumatosis intestinalis or portal vein gas or a persistent dilated loop on serial X rays, or a surgical or postmortem diagnosis.

3. Warranted treatment (including nil by mouth and antibiotics) for NEC.

Late-onset sepsis is defined as a clinical picture consistent with sepsis including initial symptoms from 48 hours after birth, and either a positive bacterial or fungal culture of blood and/or cerebrospinal fluid or a positive urine culture by sterile collection only. General guidelines for identifying positive cultures include: isolation of organisms from one blood culture and, after considering clinical/laboratory evidence, decision made to give antibiotics with therapeutic intent against this organism. Infections with coagulase-negative staphylococci and other potential contaminants or group B streptococcal antigen detected in urine should be included only if the baby is considered clinically septic and there is supporting evidence such as raised white cell count or thrombocytopenia. Viral infections must be proven by culture and/or haematological results consistent with infection. The following must not apply: mixed central nervous system or other skin flora contaminant; same blood organisms isolated from blood during the previous 14 days-repeat isolate.

We have previously applied the definitions above in our trial of placental transfusion in very preterm infants. ${ }^{34}$

The key secondary short-term end points, comprising five components of the primary composite end point, are the following:

1. Survival to hospital discharge.

2. Brain injury to 36 completed weeks.

3. ROP treated before hospital discharge by local guidelines.

4. Late-onset sepsis to 36 completed weeks.

5. NEC to 36 completed weeks.

The other secondary short-term end points are the following:

1. Time to reach full enteral feeds.

2. Number of blood transfusions to 36 completed weeks.

3. Chronic lung disease to 36 completed weeks.

4. Length of hospital stay.

5. Financial costs (for cost-effectiveness analysis in Australia only). 
The secondary long-term end point is survival at 24 to 36 months (corrected age) free from major disability. Major disability is assessed by:

1. Parent report on the Ages and Stages Questionnaire (ASQ).

2. A modified Short Health Status Questionnaire completed by a medically qualified practitioner documenting the following:

A. Major developmental delay, including language or speech problems.

B. Cerebral palsy with inability to walk unassisted at or after 2 years corrected age.

C. Severe visual loss (cannot fixate/legallyblind, or corrected acuity $<6 / 60$ in both eyes).

D. Deafness, requiring a hearing aid or cochlear implants.

3. Bayley-III Scales of Infant and Toddler Development (Bayley-III) will be collected from all infants where routinely available. A prespecified subcohort of $\sim 20 \%$ of survivors within the trial will be used to derive a cutoff score on ASQ equivalent to 2 SDs below the trial norm for cognitive scores on the Bayley-III.

Further detail on the derivation of major disability status is presented in the Statistical Analysis Plan (see e-supplemental 2).

The safety of bLF is assessed according to the relative incidence of the morbidities listed above, and the incidence of suspected adverse reactions that are classified as serious and unexpected (SUSARs). An event is considered serious if it results in death, is life-threatening (ie, the subject is at risk of death at the time of the event), requires inpatient hospitalisation or prolongation of existing hospitalisation, or results in persistent or significant disability or incapacity.

The investigator is responsible for reporting all SUSARs to the National Health and Medical Research Council (NHMRC) Clinical Trials Centre (CTC) within 1 working day of becoming aware of the event. The LIFT Trial Management Committee (TMC) and all other principal investigators participating in the study are informed of any SUSAR. The investigator or delegate at each participating institution is responsible for reporting SUSARs to the relevant ethics committee. Details of the SUSARs are reviewed by the Independent Data and Safety Monitoring Committee (IDSMC).

\section{Rationale for the composite primary end point}

Composite neonatal outcomes such as death or major morbidity, or survival without major morbidity, are frequently used in multicentre trials ${ }^{35-39}$ because parents, surviving children and clinicians place a high value on intact survival free from major morbidity. ${ }^{40}$ The primary outcome in LIFT is thus a composite of survival from enrolment to discharge free from: (1) any of three morbidities diagnosed or treated in hospital by 36 completed weeks gestational age: brain injury or late-onset sepsis or NEC; and, (2) ROP treated before hospital discharge.
Recommended approaches for constructing this primary composite outcome were followed and will be applied in analysis and interpretation of results. ${ }^{41}{ }^{42}$ Each component is clinically important (eg, the hospital morbidity end points are independently associated with later disability ${ }^{43-45}$ ), is amenable to unbiased assessment, and corresponds to biological pathways that bLF may favourably influence (eg, linked with infection and free radical disease $232546-49$ ), supporting the hypothesis that each will be affected by bLF in a similar direction. One potential concern is that not all the composite elements are equally important. Each will thus also be analysed individually as a secondary outcome, following published recommendations. ${ }^{41} 42$

\section{Sample size}

A sample size of 1500 infants has $85 \%$ power at the two-sided 5\% significance level to detect a difference in the proportion meeting the primary outcome assuming the true probability is $74 \%$ in controls and $80.5 \%$ in infants having bLF. The estimated proportion meeting the primary outcome in the control arm was informed by (1) pretrial estimates (from the Australian and New Zealand Neonatal Network), (2) a blinded (pooled) review of accumulating trial data (most recently undertaken in December 2016) and (3) the anticipated beneficial effects of the growing use of probiotics and consequent downward trend in rates of sepsis and NEC. ${ }^{50}$

\section{Actual recruitment and data collection}

A total of 1542 infants were randomised between June 2014 and September 2017. Data collection, cleaning and finalisation for the primary end point and secondary short-term end points are continuing and are anticipated to be complete by 30 April 2018. No unblinded analyses will occur until data collection and finalisation are complete.

The schedule of assessments appears in table 1. Information on in-hospital outcomes is collected via medical record review from the date of randomisation until hospital discharge. Infants transferred to other hospitals prior to discharge home are tracked by the local trial coordinator and data about the baby's care in each unit are collected to ensure that data regarding outcomes are complete. Long-term outcome data are collected in the follow-up phase up to 3 years. To facilitate good follow-up, regular contact after discharge by phone and/or post is maintained with the families and their nominated relatives and friends to confirm the family's contact details in case of a change of address.

If a parent wishes to stop the study visits, they will be asked to allow their baby's ongoing health status to be periodically reviewed either via phone contact with them or by contact with their general practitioner, or by review of their medical records or access to the national mortality registry. For parents who have been lost to follow-up in Australia, Medicare may be used to provide updated contact information and/or hospitalisations 


\begin{tabular}{|c|c|c|c|c|c|c|c|c|c|c|}
\hline & \multirow[b]{2}{*}{ Screening } & \multirow[b]{2}{*}{ Randomisation } & \multirow[b]{2}{*}{ Baseline } & \multirow{2}{*}{$\begin{array}{l}36 \\
\text { weeks }\end{array}$} & \multirow[b]{2}{*}{ Discharge } & \multicolumn{5}{|c|}{ Follow-up (months) } \\
\hline & & & & & & 6 & 12 & 18 & 24 & 36 \\
\hline Informed consent & $x$ & & & & & & & & & \\
\hline Physical assessments & & $x$ & $\mathrm{X}$ & $x$ & $x$ & & & & & \\
\hline Outcome events & & & & $\mathrm{X}$ & $X$ & & & & & \\
\hline $\begin{array}{l}\text { Parental Labour Force Participation } \\
\text { Questionnaire }\end{array}$ & & & $x$ & & & & $x$ & & $\mathrm{X}$ & $x$ \\
\hline Child hospital use & & & & & & & $x$ & & $x$ & $\mathrm{X}$ \\
\hline Ages and Stages Questionnaire & & & & & & & & & $X^{*}$ & $X^{*}$ \\
\hline
\end{tabular}

Daily treatment administration or missed doses will be recorded in the study treatment log.

Concomitant medications will not be recorded during the study, except for medications being taken when a suspected unexpected serious adverse reaction is encountered.

${ }^{*}$ Assessments will be collected at either 24 or 36 months, in line with routine follow-up scheduling for each hospital. †In a random subcohort of $\sim 20 \%$ of survivors within the trial and where routinely performed.

and the national mortality registry may be used to collect mortality information.

Deidentified trial data are prospectively recorded in the password-protected study database, developed by the NHMRC CTC, via web-based Electronic Case Report Forms (e-CRFs) completed by NICU staff in accordance with the data entry guidelines manual. Centralised data checks are performed (blinded to treatment allocation) on an ongoing basis by a data manager who oversees the resolution of any data queries raised. The investigator at each NICU confirms the accuracy of completed eCRFs.

\section{Analysis plan}

A Statistical Analysis Plan that provides additional details to the approaches described below has been finalised before any data are unblinded for analysis and is included as e-supplemental 2. All randomised subjects will be eligible for inclusion in analyses of effectiveness in accordance with the intention-to-treat analysis principle. Subjects will be analysed according to the regimen they actually received for comparisons on SUSAR rates.

The primary analysis will be a comparison between treatment groups on the proportion experiencing the primary outcome using a $\chi^{2}$ statistic that accommodates possible correlation of data between siblings from multiple births. Other binary secondary outcomes will be analysed using the same method, and comparable approaches applicable to continuous data will be applied as required. Estimates of the treatment effect adjusted for baseline characteristics will be calculated in sensitivity analyses using the relevant linear modelling approach. These modelling techniques will also be used to identify clinically important prognostic factors and to perform tests of heterogeneity in the subgroup analyses.
Hypothesis tests will be undertaken at the two-sided $5 \%$ level of significance. P-values from secondary analyses will be interpreted in proper context. Consistency of the treatment effect on the primary end point will be evaluated across the following subgroups (as specified in the relevant Cochrane review ${ }^{31}$ ): (1) birth weight $<1000 \mathrm{~g}$ and 1000-1499g; (2) randomised $\leq 72$ hours and $>72$ hours from birth; (3) those who received and did not receive probiotics by 36 completed weeks corrected gestation; (4) $\leq 28$ weeks and $>28$ completed weeks gestation at birth. ${ }^{31}$

An economic evaluation of bLF is planned for within Australia. This will combine within-trial evidence on outcomes and resource usage (eg, inpatient events/ diagnoses, length of hospitalisation, Medicare claim data (Australia only)), with external information on Australian unit costs (eg, Australian-Refined Diagnosis-Related Group). The impact of child morbidity on workforce outcomes in Australian parents will be estimated from the survey data collected at baseline, then yearly, on current employment, income and work plans.

\section{Governance, study coordination and data monitoring}

LIFT is overseen by a TMC consisting of local and international collaborators (see e-supplemental 3). A Trial Executive Committee (TEC) is selected from the TMC in order to expedite decision-making and is led by the Study Chair. The TEC meets more regularly on key scientific and/or operational issues impacting on study conduct.

LIFT is sponsored and coordinated by the NHMRC CTC. The CTC performs centralised review of study data (blinded to treatment allocation) and other documents to assess protocol compliance, data accuracy and data completeness. CTC personnel may also visit 
investigational NICUs to perform source data verification and review the investigator's NICU files and drug-handling records. By signing the informed consent form, the parent gives authorised CTC staff direct access to their child's medical records and the study data. LIFT may be subject to audit or inspection by representatives of the CTC or representatives of relevant regulatory bodies.

An Independent Data and Safety Monitoring Committee (IDSMC) monitors the progress of the study, patient safety and appropriateness of study design in accordance with a charter. It also considers other emerging evidence, including relevant RCTs and overviews of RCTs. Meeting were planned to occur once outcome data were available for the first $\mathrm{n}=550$ participants and thereafter every 12 months, or more frequently if requested by the IDSMC. The IDSMC were to advise the TMC if in their view there was proof beyond reasonable doubt of net clinical benefit or harm, for all infants or for a subset of infants that might reasonably be expected to influence the management of many clinicians. The IDSMC met in October 2016 and October 2017 and reviewed interim data on the primary end point, and on survival, against the Haybittle-Peto boundary. The IDSMC recommended continuing LIFT.

\section{Patient and public involvement}

The Miracle Babies Foundation is an Australian support and advocacy group for parents whose babies have been cared for in neonatal units. The LIFT executive worked with the Miracle Babies Foundation in developing the research question, evaluating the burden of the intervention, specifying the study design, selecting outcome measures, recruiting parents and conducting the study. The cofounder of Miracle Babies Foundation (Melinda Cruz) is furthermore an Honorary Research Associate of LIFT (see e-supplemental 3).

The results of LIFT will be communicated to participants' parents prior to publication by the investigator of the relevant site via a letter and lay summary. A summary of the results for parents, with Frequently Asked Questions, will also be placed on the NHMRC CTC website and on the Miracle Babies Foundation website. Patient advisers will be thanked in the acknowledgements of the final publication.

\section{ETHICS AND DISSEMINATION}

This study is conducted according to the Note for Guidance on Good Clinical Practice (CPMP/ICH/135/95) annotated with Therapeutic Goods Administration (TGA) comments (TGA DSEB July 2000) and in compliance with applicable laws and regulations in participating jurisdictions. The study is performed in accordance with the NHMRC Statement on Ethical Conduct in Research Involving Humans (ㄷ Commonwealth of Australia 2007) and the principles laid down by the World Medical Assembly in the Declaration of Helsinki 2008. The investigator are to comply with the protocol, except when a protocol deviation is required to eliminate immediate hazard to a subject. In this circumstance, the CTC is to be immediately advised. The study has been approved by:

- Northern Sydney Local Human Research Ethics Committee in January 2017 (Version 2.0, Reference 1003-118M).

- Australian Capital Territory Human Research Ethics Committee in March 2017 (Version 2.0, Reference ETH.6.14.140).

- Women's and Children's Health Network Human Research Ethics Committee in March 2017 (Version 2.0, Reference HREC/13/WCHN/165).

- Mercy Health Human Research Ethics Committee in June 2017 (Version 2.0, Reference R16/19).

- Northern B Health and Disability Ethics Committee in January 2018 (Version 2.0, Reference 16/NTB/12/ AM04).

Parents of potentially eligible infants are informed of the purpose of LIFT, the possible risks to participants, the voluntary nature of participation and right to withdraw and protection of confidentiality by the investigator (or designee). This is written in an information sheet (see e-supplemental 4), and evidence of informed consent is obtained in writing.

\section{Access to data and record-keeping}

The study is conducted in accordance with applicable Privacy Acts and Regulations. All information is stored securely at the NHMRC CTC, University of Sydney and is only be available to staff directly involved with the study. Access to anonymised data, and other study materials, by other parties is controlled by the TMC. All study-related documentation will be maintained for 23 years following completion of the study.

Source documents pertaining to the trial must be maintained by investigational sites. Source documents may include a subject's medical records, hospital charts, clinic charts, the investigator's subject study files, as well as the results of diagnostic tests such as X-rays, laboratory tests and ECGs. The investigator's copy of the case report forms serves as part of the investigator's record of a subject's study-related data. The following information should be entered into the subject's medical record: (1) baby's name, contact information and protocol identification; (2) the date that the baby entered the study and subject number; (3) a statement that informed consent was obtained (including the date); (4) relevant medical history; (5) results of key trial parameters; (6) occurrence of any SUSARs or outcome events; (7) the date the baby exited the study, and a notation as to whether the subject completed the study or reason for discontinuation.

\section{Dissemination policy}

A report on the results of the planned analyses described herein (and the Statistical Analysis Plan) will be prepared. The results of the primary analysis of all end points will be submitted for publication in peer-reviewed journals. The TMC will appoint a Writing Committee to draft manuscripts on behalf of the LIFT Study Group. 
Collaborators LIFT Collaborative Study Group Trial Management Committee: William Tarnow-Mordi; John Simes; Wendy Hague; Alpana Ghadge; Kei Lui; David Osborn; Girish Deshpande; Anu Kochar; Tony Lewis; Andrew Watkins; Margo Pritchard; Deborah Schofield; Abdel Latif Mohamed; Andrew Martin; Chief Investigators: William Tarnow-Mordi; Roger Soll; Paolo Manzoni; Brian Darlow; David Isaacs; Kei Lui; Deborah Schofield; Dominic Wilkinson; Andrew Martin; Wendy Hague; Associate Investigators: Alicia Wood; Anne McKenzie; Girish Despande; Hannah Verry; lan Wright; John Simes; Lisa Askie; Melinda Cruz; Nina Berry; William McGuire; Site Investigators/Coordinators: Abdel Latif Mohamed; Marg Broom; David Osoborn; Shelley Reid; John Sinn; Peggy Kwan; Mark Tracy; Carol Hua; Javeed Travadi; Ros Black; Rodney Tobiansky; Dianne Darcy/Suk Ngor Honvi Tai; Kei Lui; Jo Michalowski; Girsh Deshpande; Archana Bhaskaracharya; Tony Lewis; Emma Yeomans/Kristy Elsayed; Clare Collins; Elizabeth Noble; Pieter Koorts; Gill Lack/Lisa McKeown; Helen Liley; Wenjun Nie; Scott Morris; Kathy Cornthwaite; Anu Kochar; Louise Goodchild; Nicola Austin; Trisha Graham; Harshad Patel; Dorothy Sanchez; Independent Data Safety Monitoring Committee: Chris McKinlay; Neena Modi; Ian Marschner; Ben Stenson; David Espinoza (unblinded statistician, nonvoting).

Contributors WT-M, KL, AM and PM conceived of and designed the study. WT-M, AM, PM, AG and KL acquired the funding. AG and RB coordinated the implementation of the study and the acquisition of data. AM and WT-M drafted the manuscript. All authors (AM, AG, PM, KL, RB, WT-M) revised the manuscript critically for important intellectual content and approved the final version.

Funding This work is supported by the National Health and Medical Research Council (grant no: APP1047100).

Competing interests None declared.

Patient consent Not required.

Ethics approval Northern Sydney Local Human Research Ethics Committee in January 2017 (Version 2.0, Reference 1003-118M); Australian Capital Territory Human Research Ethics Committee in March 2017 (Version 2.0, Reference ETH.6.14.140); Women's and Children's Health Network Human Research Ethics Committee in March 2017 (Version 2.0, Reference HREC/13/WCHN/165); Mercy Health Human Research Ethics Committee in June 2017 (Version 2.0, Reference R16/19); Northern B Health and Disability Ethics Committee in January 2018 (Version 2.0, Reference 16/NTB/12/AM04).

Provenance and peer review Not commissioned; externally peer reviewed.

Open access This is an open access article distributed in accordance with the Creative Commons Attribution Non Commercial (CC BY-NC 4.0) license, which permits others to distribute, remix, adapt, build upon this work non-commercially, and license their derivative works on different terms, provided the original work is properly cited, appropriate credit is given, any changes made indicated, and the use is non-commercial. See: http://creativecommons.org/licenses/by-nc/4.0/.

\section{REFERENCES}

1. Blencowe $\mathrm{H}$, Cousens $\mathrm{S}$, Oestergaard $\mathrm{MZ}$, et al. National, regional, and worldwide estimates of preterm birth rates in the year 2010 with time trends since 1990 for selected countries: a systematic analysis and implications. Lancet 2012;379:2162-72.

2. Hamilton BE, Martin JA, Ventura SJ. Births: preliminary data for 2012. Natl Vital Stat Rep 2013;62:1-20.

3. Chow SSW, Le Marsney R, Creighton P, et al. Report of the Australian and New Zealand Neonatal Network 2015. Sydney: ANZNN, 2017.

4. Australian Institute of Health and Welfare. Australia's mothers and babies 2015-in brief. Perinatal statistics series no. 33 Cat no. PER 91. Canberra: Australian Institute of Health and Welfare, 2017.

5. Saigal S, Doyle LW. An overview of mortality and sequelae of preterm birth from infancy to adulthood. Lancet 2008;371:261-9.

6. Moore T, Hennessy EM, Myles J, et al. Neurological and developmental outcome in extremely preterm children born in England in 1995 and 2006: the EPICure studies. BMJ 2012;345:e7961.

7. Botting N, Powls A, Cooke RW, et al. Attention deficit hyperactivity disorders and other psychiatric outcomes in very low birthweight children at 12 years. J Child Psychol Psychiatry 1997;38:931-41.

8. Svedenkrans J, Henckel E, Kowalski J, et al. Long-term impact of preterm birth on exercise capacity in healthy young men: a national population-based cohort study. PLoS One 2013;8:e80869.

9. Lönnerdal B. Nutritional roles of lactoferrin. Curr Opin Clin Nutr Metab Care 2009;12:293-7.

10. Scott PH. Plasma lactoferrin levels in newborn preterm infants: effect of infection. Ann Clin Biochem 1989;26(Pt 5):412-5.
11. Decembrino L, DeAmici M, De Silvestri $A$, et al. Plasma lactoferrin levels in newborn preterm infants with sepsis. J Matern Fetal Neonatal Med 2017;30:2890-3.

12. Ochoa TJ, Cleary TG. Effect of lactoferrin on enteric pathogens. Biochimie 2009;91:30-4.

13. Valenti $P$, Antonini G. Lactoferrin: an important host defence against microbial and viral attack. Cell Mol Life Sci 2005;62:2576-87.

14. Leitch EC, Willcox MD. Lactoferrin increases the susceptibility of $\mathrm{S}$. epidermidis biofilms to lysozyme and vancomycin. Curr Eye Res 1999;19:12-19.

15. Groot F, Geijtenbeek TB, Sanders RW, et al. Lactoferrin prevents dendritic cell-mediated human immunodeficiency virus type 1 transmission by blocking the DC-SIGN--gp120 interaction. J Virol 2005;79:3009-15.

16. Lupetti A, Paulusma-Annema A, Welling MM, et al. Candidacidal activities of human lactoferrin peptides derived from the $\mathrm{N}$ terminus. Antimicrob Agents Chemother 2000;44:3257-63.

17. Rahman MM, Kim WS, Ito T, et al. Growth promotion and cell binding ability of bovine lactoferrin to Bifidobacterium longum. Anaerobe 2009;15:133-7.

18. Kaufman DA. Lactoferrin supplementation to prevent nosocomial infections in preterm infants. JAMA 2009;302:1467-8.

19. Vorland LH, Ulvatne $\mathrm{H}$, Rekdal $\mathrm{O}$, et al. Initial binding sites of antimicrobial peptides in Staphylococcus aureus and Escherichia coli. Scand J Infect Dis 1999;31:467-73.

20. Vorland LH, Ulvatne $\mathrm{H}$, Andersen J, et al. Antibacterial effects of lactoferricin B. Scand J Infect Dis 1999;31:179-84.

21. Manzoni P, García Sánchez R, Meyer M, et al. Exposure to gastric acid inhibitors increases the risk of infection in preterm very low birth weight infants but concomitant administration of lactoferrin counteracts this effect. J Pediatr 2018;193:62-7.

22. Embleton NE, Pang N, Cooke RJ. Postnatal malnutrition and growth retardation: an inevitable consequence of current recommendations in preterm infants? Pediatrics 2001;107:270-3.

23. Venkatesh M, Abrams S. Can lactoferrin prevent neonatal sepsis and necrotizing enterocolitis? Expert Rev Anti Infect Ther 2009;7:515-25.

24. AlFaleh K, Anabrees J. Probiotics for prevention of necrotizing enterocolitis in preterm infants. Cochrane Database Syst Rev 2014;(4):CD005496.

25. Raghuveer TS, McGuire EM, Martin SM, et al. Lactoferrin in the preterm infants' diet attenuates iron-induced oxidation products. Pediatr Res 2002;52:964-72.

26. Office of Food Additive Safety. GRAS notice GRN 669. Food and Drug Administration. Generally Recognized As Safe (GRAS) Notice: The Use ofBovine Milk-derived Lactoferrin In Term Milk-based Infant Formulas and Toddler Formulas. https://www.fda.gov/downloads/ food/ingredientspackaginglabeling/gras/noticeinventory/ucm592102. pdf (accessed 5 Mar 2018).

27. Cheng JB, Wang JQ, Bu DP, Dp B, et al. Factors affecting the lactoferrin concentration in bovine milk. J Dairy Sci 2008;91:970-6.

28. Yamauchi K, Toida T, Nishimura S, et al. 13-Week oral repeated administration toxicity study of bovine lactoferrin in rats. Food Chem Toxicol 2000;38:503-12.

29. Manzoni P, Rinaldi M, Cattani S, et al. Bovine lactoferrin supplementation for prevention of late-onset sepsis in very low-birthweight neonates: a randomized trial. JAMA 2009;302:1421-8.

30. Pammi M, Abrams SA. Oral lactoferrin for the prevention of sepsis and necrotizing enterocolitis in preterm infants. Cochrane Database Syst Rev 2011;(10):CD007137.

31. Pammi M, Suresh G. Enteral lactoferrin supplementation for prevention of sepsis and necrotizing enterocolitis in preterm infants. Cochrane Database Syst Rev 2017;(6):CD007137.

32. ANZNN. Australian and New Zealand Neonatal Network. ANZNN 2009 Data Dictionary. Sydney, Australia. Sydney: UNSW, 2008.

33. ANZNN. Australian and New Zealand Neonatal Network. ANZNN 2009/2010 Follow-up Data Dictionary. Sydney, Australia.: UNSW, 2013.

34. Tarnow-Mordi W, Morris J, Kirby A, et al. Delayed versus Immediate Cord Clamping in Preterm Infants. N Engl J Med 2017;377:2445-55.

35. Askie LM, Henderson-Smart DJ, Irwig L, et al. Oxygen-saturation targets and outcomes in extremely preterm infants. $N$ Engl J Med 2003;349:959-67.

36. Askie LM, Brocklehurst P, Darlow BA, et al. NeOProM: Neonatal Oxygenation Prospective Meta-analysis Collaboration study protocol. BMC Pediatr 2011;11:6.

37. Morley CJ, Davis PG, Doyle LW, et al. Nasal CPAP or intubation at birth for very preterm infants. N Engl J Med 2008;358:700-8.

38. Jacobs SE, Morley CJ, Inder TE, et al. Whole-body hypothermia for term and near-term newborns with hypoxic-ischemic encephalopathy: a randomized controlled trial. Arch Pediatr Adolesc Med 2011;165:692-700. 
39. Schmidt B. Methylxanthine therapy for apnea of prematurity: evaluation of treatment benefits and risks at age 5 years in the international Caffeine for Apnea of Prematurity (CAP) trial. Biol Neonate 2005;88:208-13.

40. Saigal S, Stoskopf BL, Feeny D, et al. Differences in preferences for neonatal outcomes among health care professionals, parents, and adolescents. JAMA 1999;281:1991-7.

41. Cordoba G, Schwartz L, Woloshin S, et al. Definition, reporting, and interpretation of composite outcomes in clinical trials: systematic review. BMJ 2010;341:c3920.

42. Ferreira-González I, Permanyer-Miralda G, Busse JW, et al. Methodologic discussions for using and interpreting composite endpoints are limited, but still identify major concerns. J Clin Epidemiol 2007;60:651-7. discussion 658-662.

43. Schmidt B, Asztalos EV, Roberts RS, et al. Impact of bronchopulmonary dysplasia, brain injury, and severe retinopathy on the outcome of extremely low-birth-weight infants at 18 months: results from the trial of indomethacin prophylaxis in preterms. JAMA 2003;289:1124-9.

44. Bassler D, Stoll BJ, Schmidt B, et al. Using a count of neonatal morbidities to predict poor outcome in extremely low birth weight infants: added role of neonatal infection. Pediatrics 2009;123:313-8.

45. Schulzke SM, Deshpande GC, Patole SK. Neurodevelopmental outcomes of very low-birth-weight infants with necrotizing enterocolitis: a systematic review of observational studies. Arch Pediatr Adolesc Med 2007;161:583-90.

46. Actor JK, Hwang SA, Kruzel ML. Lactoferrin as a natural immune modulator. Curr Pharm Des 2009;15:1956-73.

47. Berlutti F, Schippa S, Morea C, et al. Lactoferrin downregulates pro-inflammatory cytokines upexpressed in intestinal epithelial cells infected with invasive or noninvasive Escherichia coli strains. Biochem Cell Biol 2006;84:351-7.

48. Kapoor K, Basu S, Das BK, et al. Lipid peroxidation and antioxidants in neonatal septicemia. J Trop Pediatr 2006;52:372-5.

49. Silveira RC, Procianoy RS, Dill JC, et al. Periventricular leukomalacia in very low birth weight preterm neonates with high risk for neonatal sepsis. J Pediatr 2008;84:211-6.

50. Deshpande GC, Rao SC, Keil AD, et al. Evidence-based guidelines for use of probiotics in preterm neonates. BMC Med 2011;9:92. 